\title{
Trans-oral penetrating trauma to the neck: the innocuous pen cap and the value of CT Scan
}

\author{
M Nathie, ${ }^{1}$ S Maharaj ${ }^{2}$ \\ ${ }^{1}$ University of the Witwatersrand, Johannesburg, South Africa \\ ${ }^{2}$ Department of Otorhinolaryngology, University of the Witwatersrand, Johannesburg, South Africa
}

Corresponding author: Dr Shivesh Maharaj (shivesh.maharaj@wits.ac.za)

\begin{abstract}
Summary: A significant number of otorhinolaryngological emergency visits are caused by foreign bodies, and occasionally they can result in life-threatening injury. This report highlights the value of neck CT in the evaluation of suspected radiolucent foreign bodies penetrating the neck from the oral cavity. This guided the surgical approach to remove the foreign body safely without complication.
\end{abstract}

Keywords: Trans-oral penetrating injury, deep neck space, pen cap, Computer Tomography

S Afr J Surg 2019;57(3)

http://dx.doi.org/10.17159/2078-5151/2019/v57n3a2912

\section{Case presentation}

A six-year-old child, accompanied by the parents, presented to the Charlotte Maxeke Johannesburg Academic Hospital (CMJAH) casualty with a one-day history of playing in a moving vehicle, with a pen in his mouth. The vehicle came to an abrupt stop and the pen cap penetrated the soft tissues of the oral cavity into the neck. The child was fully awake and responsive. There was no active bleeding and the child was haemodynamically stable. The child was breathing with ease. Clinical examination of the head and neck region revealed a swelling posterior to left ear, and anterior to mastoid process. It was tender to palpation. Intra-oral examination revealed a small mucosal injury $(<0.5 \mathrm{~cm})$ between the uvula and the left tonsil. Flexible nasopharyngoscopy confirmed the airway to be patent, and no foreign bodies or other abnormality was seen . X-rays of the neck showed only soft tissue swelling. In view of the soft tissue swelling and the lack of a radiopaque abnormality on a neck radiograph, a CT Scan from the base of skull to clavicle was obtained.

The CT scan (Figure 1a and b) showed an ovoid shape foreign body projected along the left carotid and parapharyngeal spaces bounded medially by $\mathrm{C} 2$ vertebra, laterally by the left sternocleidomastoid muscle and posteriorly by the body of the mandible.

There was extensive surgical emphysema involving the left retropharyngeal, parapharyngeal, carotid and prevertebral spaces and extending into the superior mediastinum. There was no attenuation of the airway.

The left common carotid artery, external carotid artery and internal carotid artery were normal in calibre, with no extravasation of contrast. The left internal jugular vein was attenuated due to surgical emphysema.
A modified neck exploration was conducted, where the skin incision $( \pm 3 \mathrm{~cm})$ was made directly at the most pointing area. The foreign body, identified as a pen cap was removed with no complications (Figure 1c). His postoperative recovery was uneventful, and the patient was discharged 72 hours later.

\section{Discussion}

Patients with suspected foreign bodies are common in childhood otorhinolaryngological emergency departments. The majority of foreign bodies in the aerodigestive tract are either inhaled or ingested objects. ${ }^{1-3}$ Palatal lacerations are a common insult due to transoral penetrating foreign bodies, especially in the paediatric population who place objects in their mouth. ${ }^{4}$ In the oral region, the anatomical location of the injury is generally easily identified by clinical examination without the aid of specialised radiological investigation, and removal is by direct extraction. Fatal complications of transoral penetrating foreign bodies do occur in rare cases, these include carotid trauma, or thrombosis, intracranial injuries, mediastinitis or airway obstruction. ${ }^{5-8}$ Similar to those earlier reported cases, our patient's initial history accompanied by corroboration by an adult eye-witness provided a rather misleading impression as to a harmless trauma. He posed a diagnostic dilemma requiring further imaging in order to ascertain the trajectory and location of the suspected foreign body in order to undergo safe surgical removal. Given the trajectory that the pen cap had passed between the carotid artery and internal jugular vein it was particularly fortuitous that the child did not present in extremis with a vascular injury or airway compromise. 


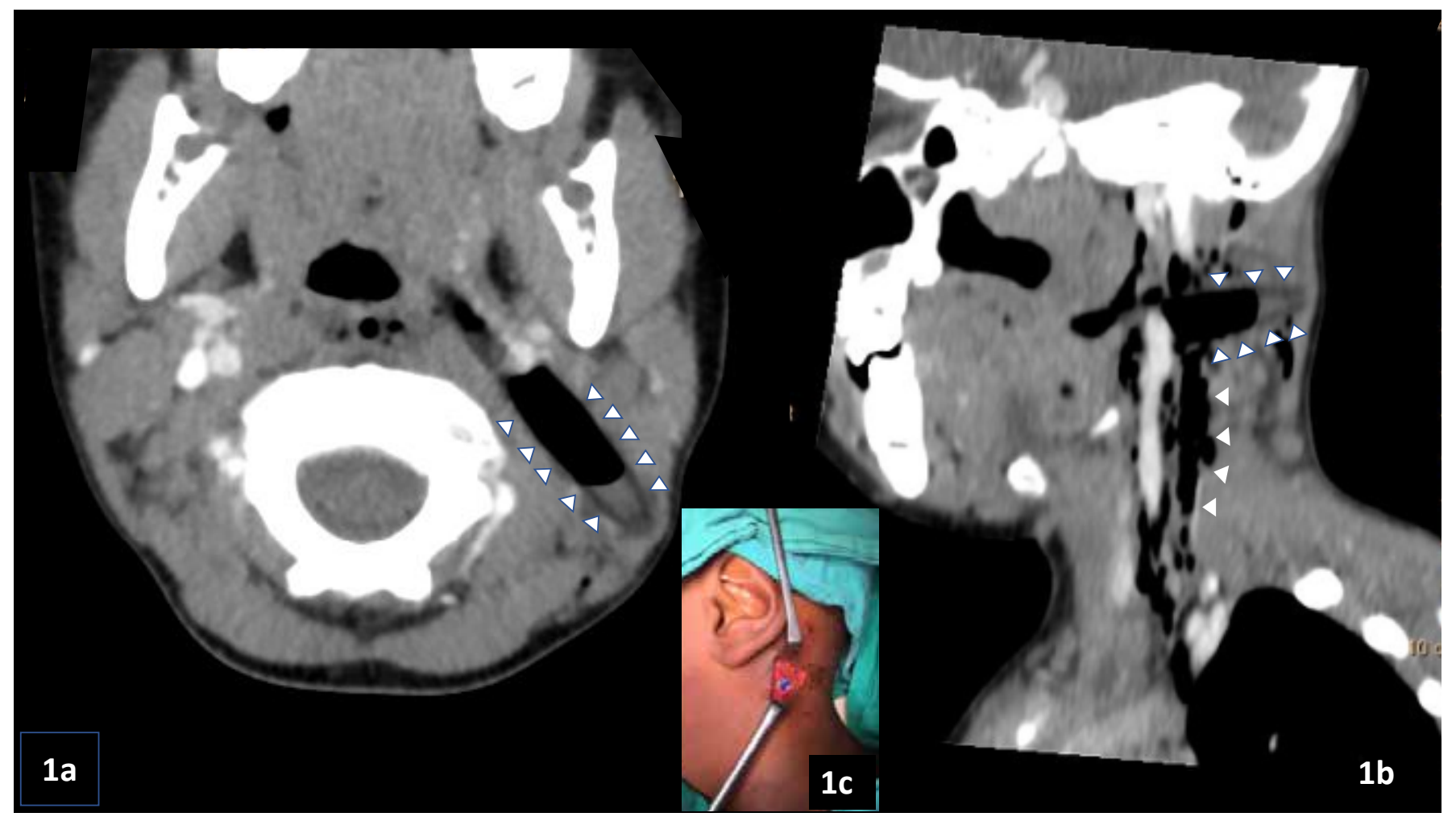

Figure 1

a) Axial CT scan of the neck showing pen cap tract (white arrows blue surrounds) and air in the deep neck spaces

b) Sagittal CT scan showing pen cap (white arrows blue surrounds) and soft tissue air spaces (white arrows)

c) Intraoperative picture, blue pen cap seen between retractors

\section{Conclusion}

History or clinical examination alone may be inadequate to raise the suspicion of a retained foreign body in the deep neck spaces in the situation of trans-oral penetrating injury of the oropharyngeal cavity. Computer Tomography is valuable for the evaluation of trans-oral trauma to the oropharynx, especially because it could reveal the presence of a retained foreign body in the deep neck spaces and the proximity of vital structures, thus guiding a surgical approach and enabling safe minimally invasive removal of the foreign body. We are also alerted to the potential dangers of the innocuous pen cap.

\section{Acknowledgements}

Department of Radiology, Charlotte Maxeke Johannesburg Academic Hospital, University of the Witwatersrand

\section{REFERENCES}

1. Kim SY, Park B, Kong IG, et al. Analysis of ingested foreign bodies by age, type and location: a retrospective observational study. Clin Otolaryngol. $2016 ; 41(6): 640-5$.

2. Blanco Ramos M, Botana-Rial M, Garcia-Fontan E, et al. Update in the extraction of airway foreign bodies in adults. $\mathrm{J}$ Thoracic Dis. 2016;8(11):3452-6.

3. Berdan EA, Sato TT. Paediatric airway and oesophageal foreign bodies. Surg Clin North Am. 2017;(97):85-91.

4. Radkowski D, McGill TJ, Healy GB, et al. Penetrating trauma of the oropharynx in children. Laryngoscope. 1993;103:991-4.

5. Randall DA, Kang DR. Current management of penetrating injuries of the soft palate. Otolaryngol Head Neck Surg. 2006;135(3):356-60.

6. Sasaki T, Toriumi S, Asakage T, et al. The toothbrush: a rare but potentially life-threatening cause of penetrating oropharyngeal trauma in children. Pediatrics. 2006;118:1284-6.

7. Samuthrat $\mathrm{T}$, Ye K, Tong Y. Transoral Intracranial Injury via Middle Skull Base by Blunt Chopstick in a Child. World Neurosurg. 2017;103:952.e11-952.e17.

8. Law RC, Fouque CA, Waddell A, et al. Lesson of the week. Penetrating intra-oral trauma in children. BMJ. 1997;314:50-1. 